\title{
Loss of the associated $\alpha$-particles in the tagged neutron generators
}

\author{
D. Sudac ${ }^{\text {a,* }}$, K. Nad ${ }^{a}$, J. Obhodas ${ }^{\text {a }}$, V.M. Bystritsky ${ }^{b}$, V. Valkovic ${ }^{c, 1}$ \\ ${ }^{a}$ Institute Ruder Boskovic, P.O. Box 180, 10002 Zagreb, Croatia \\ ${ }^{\mathrm{b}}$ Joint Institute for Nuclear Research, Moscow region, Dubna 141 980, Russia \\ ' Kvinticka 62, 10000 Zagreb, Croatia
}

\section{A B S T R A C T}

The reported loss of $\alpha$-particles in the $14 \mathrm{MeV}$ tagged neutron generators has been investigated using two neutron generators equipped with $\alpha$-particle counters and two neutron detectors. One neutron detector was put right in the middle of the tagged neutron cone and another one was put outside the cone. By measuring the difference between double (neutron-neutron) and triple ( $\alpha$-neutron-neutron) coincidences it is possible to deduce the $\alpha-$ particle loss since the number of triple coincidences should be equal to the number of double coincidences. In all measurements performed a deficit of triple with respect to double coincidences has been observed. This deficit was smallest for the threshold of $\alpha$-particle Constant Fraction Discriminator $(\alpha \mathrm{CFD}$ ) being 0 and maximum allowed voltage of $\alpha$-particle detector being $-1.7 \mathrm{kV}$. The smallest measured deficit value was equal to $13 \pm 1 \%$. From the observed results it was concluded that the deficit was due to a number of non-detected $\alpha$-particles that loose sufficient quantity of energy while traveling to the detector because of collisions with particles present in the neutron tube and/or in the tritium target. These $\alpha$-particles will not be detected as they fall under the threshold of $\alpha$ CFD discriminator. Magnetic fields present in the system worsen the situation since they are forcing $\alpha$ particles to travel larger distances because of toroidal movement and undergoing additional collisions. Tagged neutron technique has many kind of applications and it is particularly important for high accuracy nuclear cross-sections measurements when $\alpha$-particles losses must be carefully assessed.

\section{Introduction}

The $14 \mathrm{MeV}$ neutrons are produced by using $\mathrm{d}+\mathrm{t} \rightarrow \mathrm{n}+\alpha$ reaction $(Q=17.590 \mathrm{MeV})$ having a pronounced peak in the cross section for deuteron bombarding energy around $100 \mathrm{keV}$. Usually deuteron beam is impinging on TiT target resulting in isotropic emission of neutrons in the center of mass.

The detection of neutron beam and associated $\alpha$-particles $\left(E_{\alpha}=3.5 \mathrm{MeV}\right)$ emitted in a corresponding solid angle defines the so-called "tagged neutron beam" which allows time-of-flight analysis and significant electronic reduction of background in the studies of the neutron induced reactions [1]. The "tagged" neutrons interact with the interrogated object and can produce $\gamma$-radiation and/or neutron radiation by allowing the non-destructive chemical analysis of the interrogated object at the position of interest, usually with $5 \mathrm{~cm}$ space resolution. This approach is typically used for material identification in neutron screening systems developed

\footnotetext{
* Corresponding author.

E-mail address: valkovic@irb.hr (V. Valkovic).

${ }^{1}$ Tel.: +3851468 0101; fax: +38514680239.
}

recently for passenger luggage [2], airplane [3] and sea containers $[4,5]$ screening.

For most of the $14 \mathrm{MeV}$ neutron applications, and especially for cross sections determination, it is necessary to make a neutron flux measurement. In addition, the neutron flux has to be monitored due to tritium consumption over the time. For the tagged neutron accelerators it is assumed that number of neutrons could be deduced from the counting rate of the $\alpha$-particle detector after the correction for the geometrical efficiency. However, in some experiments performed with triple ( $\alpha$-neutron-neutron) and double (neutron-neutron) coincidences, the $\alpha$-particle deficit was noted. The experiment in which $15 \%$ of the alphas were not counted had a set-up including a plastic scintillator located in the cone of the central $\alpha$-detector pixel and another plastic scintillator located about $50 \mathrm{~cm}$ away from the first detector and at the $45^{\circ}$ angle with respect to the line between the neutron production spot in the generator and the first detector. Simple kinematics of elastic neutron-proton scattering shows that at the angle of $45^{\circ}$, the neutrons arriving at the second detector should have energy of the $7 \mathrm{MeV}$. By measuring the triple coincidences between the alpha pulse and the detection of neutron in each of the detectors, the coincidence rate between detectors for which there was an alpha pulse was determined. The double coincidences rate included coincidences between the two neutron detectors only. The 
ratio of the triple coincidence rate to the double coincidence rate was 0.85 .

The same measurements were repeated by Mihalczo at al. [6]. However, as the neutron source they used two completely different designs of API $\mathrm{d}+\mathrm{t}$ generators produced by Thermo Fischer; one was API-120 neutron generator and another one was produced in 1990s and contained focusing lens. Both neutron generators had alpha particle detectors made of thin $\mathrm{ZnO}$ coating, over-coated with a very thin layer of aluminum to stop scattered deuterons, tritons and light produced on the fiber optic face plate. The results for the ratio of the triple to double coincidence rate were $86 \%$ and $85 \%$, respectively.

The above described experiments showed 14\%-15\% deficit in alpha particles, counted independently of the type of generator or $\alpha$-particle detector used. We decided to look into this problem with two different experimental set-ups using API-120 and $300 \mathrm{keV}$ Cockcroft-Walton accelerator, respectively. The results are showed in this paper.

\section{Experimental set-up}

The experimental set-up with API-120 neutron source is shown in Fig. 1. The neutron source API-120 (acquired from ThermoElectron) was equipped with $\operatorname{YAP}(\mathrm{Ce}) \alpha$-particle detector with crystal thickness of $0.5 \mathrm{~mm}$ and diameter of $19 \mathrm{~mm}$. One side of the crystal is coated with $1 \mathrm{mg} / \mathrm{cm}^{2}$ of silver in order to avoid impact of low energy particles (mainly electrons). Crystal is attached to inner side of the fiber optic plate (FOP) $7.5 \mathrm{~cm}$ distant from tritium target, while the photomultiplier tube (PMT) was attached at the outer side of FOP. YAP(Ce) with FOP and PMT are the components of the alpha particle detector assembly.
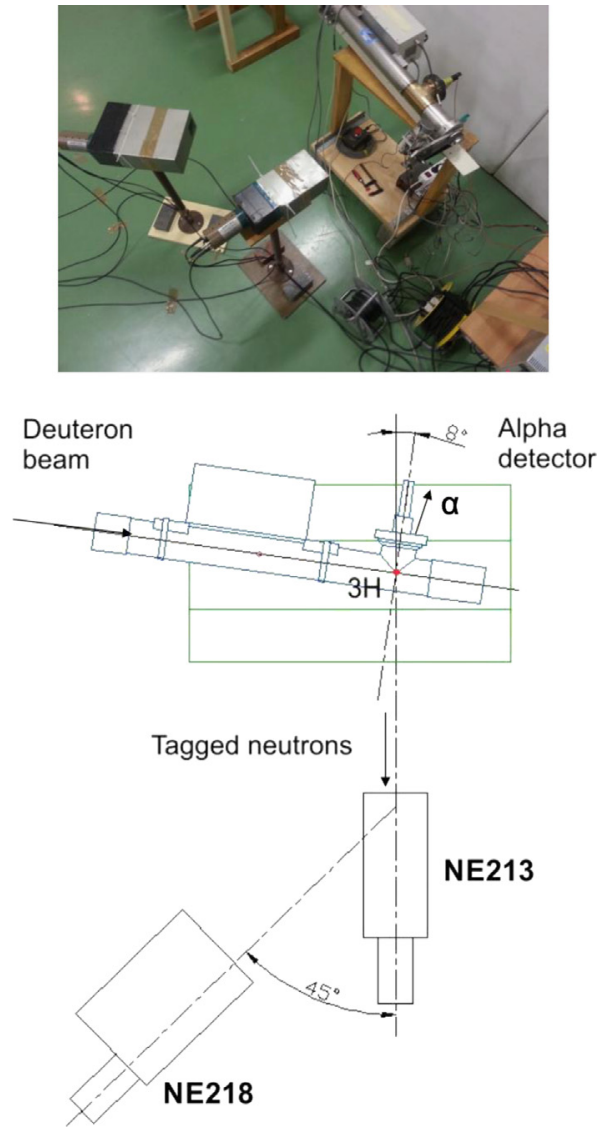

Fig. 1. Experimental set-up with API-120.
The axis of the neutron accelerator tube, along which the deuterons are accelerated, has been inclined for $8^{\circ}$ in order to place the neutron detector, the 3 “ $\times 3$ " NE213, precisely inside the cone of tagged neutrons at the position of neutrons maximum intensity (Fig. 1). The second neutron detector, the 3 “ $\times 3$ ” NE218, was placed outside the tagged neutron cone under an angle of $45^{\circ}$ with respect to the cone axis. The distance between two neutron detectors was $50 \mathrm{~cm}$, the same as the distance between detector NE213 and tritium target inside the API- 120.

Experimental set-up on Cockcroft-Walton accelerator is shown in Fig. 2. At the end of the beam line there is a tritium target position. The alpha detector, the $\mathrm{YAP}(\mathrm{Ce})$, has crystal thickness of $1 \mathrm{~mm}$ and diameter of $19 \mathrm{~mm}$. It was placed under the angle of $53^{\circ}$ with respect to the incident deuteron beam at the distance $d=21 \mathrm{~cm}$ from tritium target. The crystal which is attached on safire window placed in CF63 flange has the PMT Hamamatsu R1450. The first neutron detector, the 3 " $\times 3$ " NE213, was placed at the distance of $160 \mathrm{~cm}$ from the tritium target. At this distance the horizontal tagged neutron beam radius (FWHM) is $16 \pm 3 \mathrm{~cm}$, as shown in Fig. 4. The vertical profile has similar shape. The second neutron detector, the 3 " $\times 3$ " NE218, was placed outside the cone of tagged neutron beam under the angle of $45^{\circ}$ with respect to cone axis, $50 \mathrm{~cm}$ distant from the detector NE213 (as in set-up with API-120). The deuteron beam energy was $100 \mathrm{keV}$.

The electronics set-up used in both measurements are shown in Fig. 3. The NE213 output pulse was a START pulse for nnTAC, measuring coincidences between two neutron detectors and it was also a START pulse for $\alpha$-TAC, measuring the coincidences between NE213 and alpha detectors. SCA outputs from both TACS were taken to an AND gate generating trigger signal for the computer A. Output from nnTAC was split into the computer A, being triggered by triple coincidences and computer $\mathrm{B}$ triggered by double coincidences. Double coincidences were coincidences between two neutron detectors; triple coincidences were coincidences between two neutron detectors and an alpha detector. Each computer had ADC card with four inputs so four signals were measured simultaneously by each computer: output from nnTAC, output from $\alpha$-particle detector, output from NE213 and output
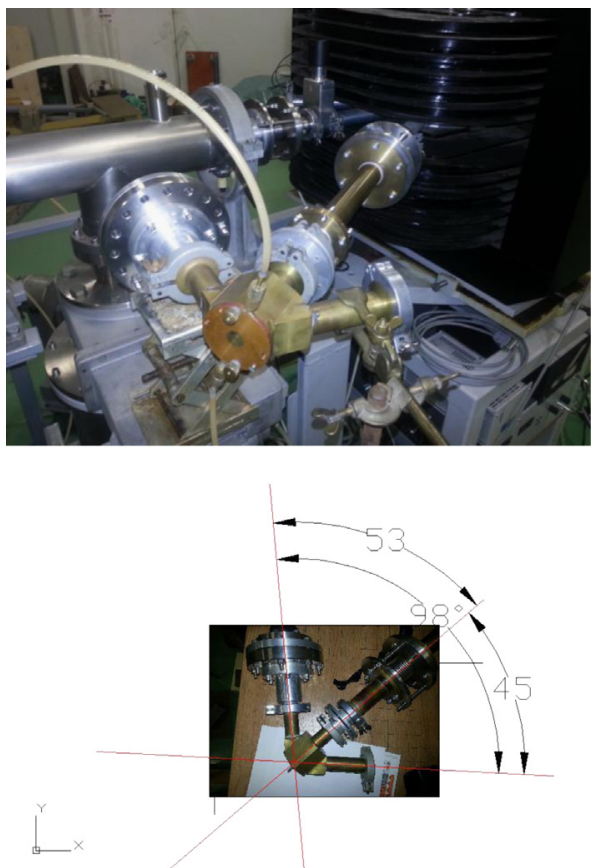

Fig. 2. End of Cockcroft-Walton accelerator beam line with water cooled tritium target and associated alpha detector chamber. 


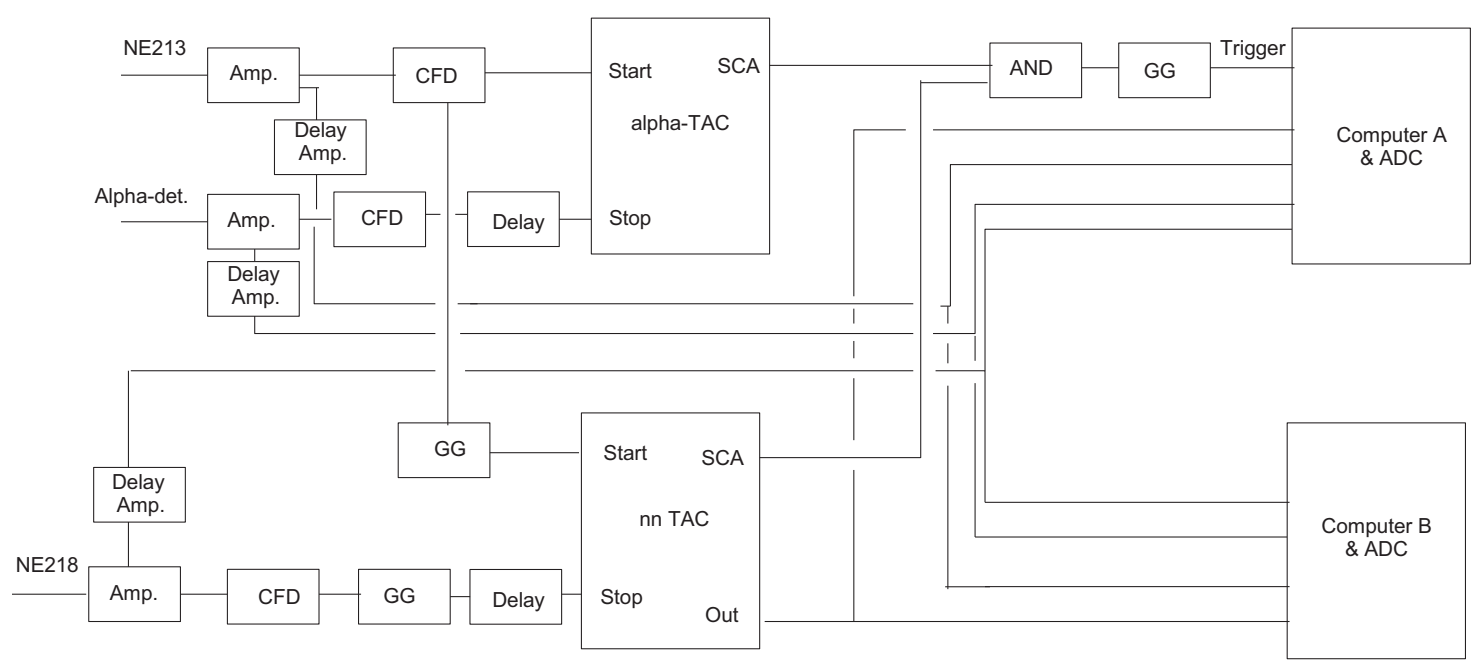

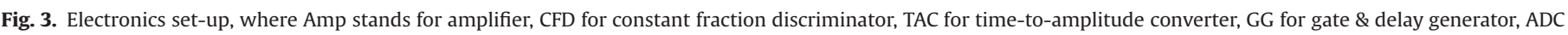
for analog-to-digital converter.

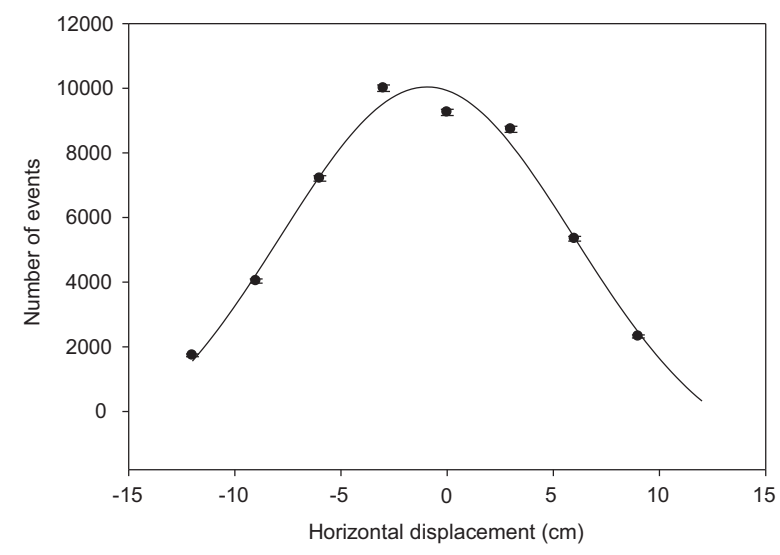

Fig. 4. Horizontal profile of tagged neutron beam, experimental point and best fit curve are shown.

from NE218. Neutron intensity was kept fixed in all measurements with API $120\left(10^{7} \mathrm{n} / \mathrm{s}\right.$ in $\left.4 \pi\right)$. The neutron beam intensity obtained with Cockcroft-Walton accelerator $(\mathrm{CW})$ was $<10^{7} \mathrm{n} / \mathrm{s}$ in $4 \pi$. Due to set-up geometry used in CW experiment in which NE213 was $160 \mathrm{~cm}$ apart from the neutron source, the neutron detectors counted much less when compared with API 120 experiment.

\section{Results of the measurements}

\subsection{Measurements with API-120}

Fig. 5 shows time spectra of events detected in two neutron detectors-the nnTAC output recorded on computer B measuring $\gamma-\gamma$ and $n-n$ coincidences. Fig. 5a presents the $\gamma \gamma$ peak from the scattering of ${ }^{60} \mathrm{Co}$ gamma rays. The ${ }^{60} \mathrm{Co}$ source was placed on top of NE213 detector. Gamma rays from ${ }^{60} \mathrm{Co}$ having average energy of $1.25 \mathrm{MeV}$ were scatter at an angle of $90^{\circ}$ into the second detector, NE218. Energy of ${ }^{60} \mathrm{Co}$ Compton gamma ray scattering deposited in NE218 for this angle is $\approx 0.9 \mathrm{MeV}$. When the neutron generator was used instead of ${ }^{60} \mathrm{Co}$ radioactive source, the time spectrum of double coincidences had two peaks (Fig. 5b). The first peak corresponds to $\gamma-\gamma$ coincidences which are determined by its position in time spectrum, while the second peak corresponds to $\mathrm{n}-\mathrm{n}$ coincidences, i.e. to the neutrons of energy $14 \mathrm{MeV}$ scattered
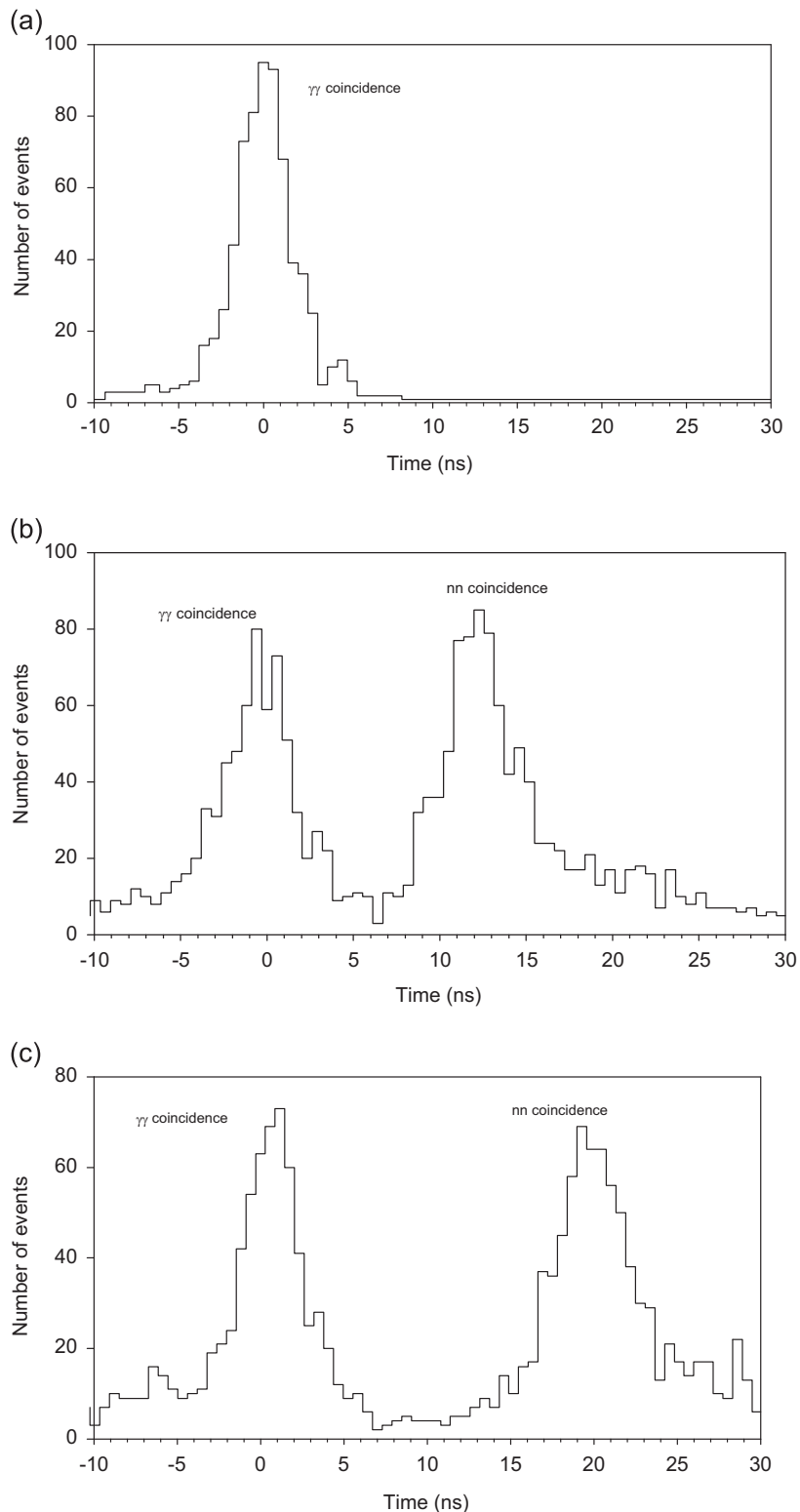

Fig. 5. Time spectra of events detected in two neutron detectors, nnTAC output. 


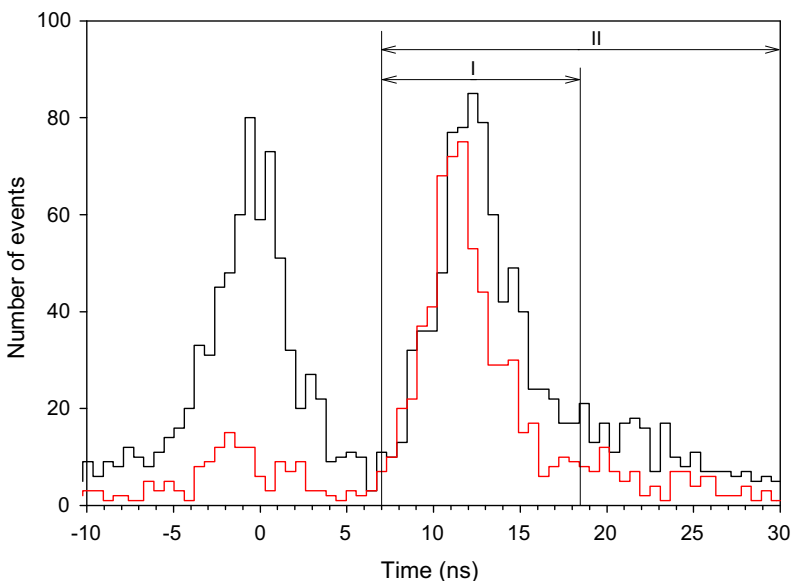

Fig. 6. Time spectra for double (black) and triple (red) coincidences. (For interpretation of the references to color in this figure legend, the reader is referred to the web version of this article.)

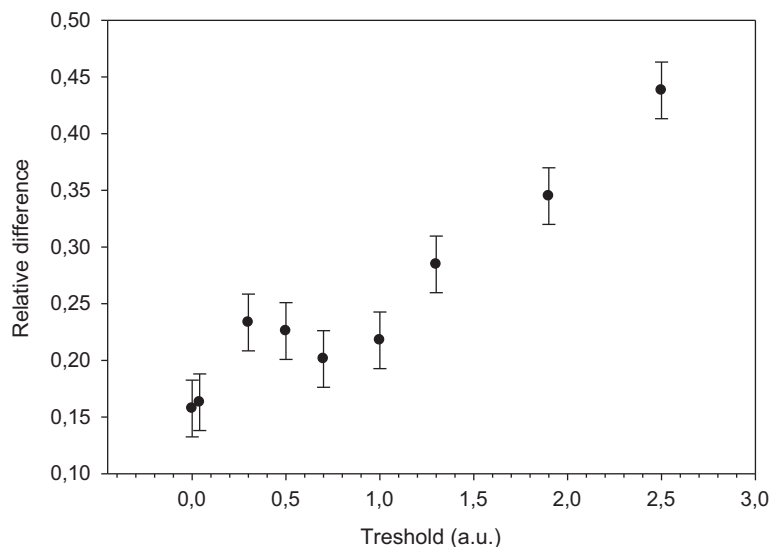

Fig. 7. The relative difference between the number of double and triple coincidences as a function of threshold value on $\alpha$ CFD. The HV on alpha detector is $-1.6 \mathrm{kV}$. Number of events was summed over the channels inside the time window "I". Random coincidences were subtracted.

inside detector NE213 at an angle of $45^{\circ}$ into the second detector, the NE218, with the energy of $7 \mathrm{MeV}$. The time difference between two peaks corresponds to the time-of-flight of $7 \mathrm{MeV}$ neutrons. When distance between two neutron detectors was increased by $30 \mathrm{~cm}$, the separation between two peaks increased as well (Fig. 5c). One can also observe the $1 \mathrm{~ns}$ shift of the $\gamma-\gamma$ peak corresponding to the additional distance of $30 \mathrm{~cm}$ that gamma rays have had to travel.

Fig. 6 shows the time spectra for double (black) and triples (red) coincidences. The number of events in $n-n$ peak should be the same in both cases. However, there is an obvious deficit of events in $n-n$ peak in the case of the triple coincidences with respect to the number of double coincidences. This deficit depends on the size of integration window (window I and II). In addition, the deficit in $n-n$ peak depends on the threshold of $\alpha$ CFD and high voltage applied to the $\alpha$-detector. The smallest relative difference ((double-triple)/ double) is for zero threshold (Fig. 7) and maximum allowed voltage on PMT (Fig. 8). In Fig. 6 the alpha detector HV was $-1.6 \mathrm{kV}$, while the $\alpha$ CFD threshold was 0.7 (a.u.). Fig. 9 shows the neutron-gated $\alpha$-particle pulse height distribution for $-1.6 \mathrm{kV}$ HV and zero threshold. The red part of spectrum came in time later than the black part (Fig. 10) indicating the presence of lower energy $\alpha$-particles $(\sim 2.9 \mathrm{MeV})$ compared to the $3.5 \mathrm{MeV} \alpha$-particles produced at an angle of 90 . The possible effect of magnetic field has been investigated by placing a small permanent magnet $(0.1 \mathrm{~T})$ at three different

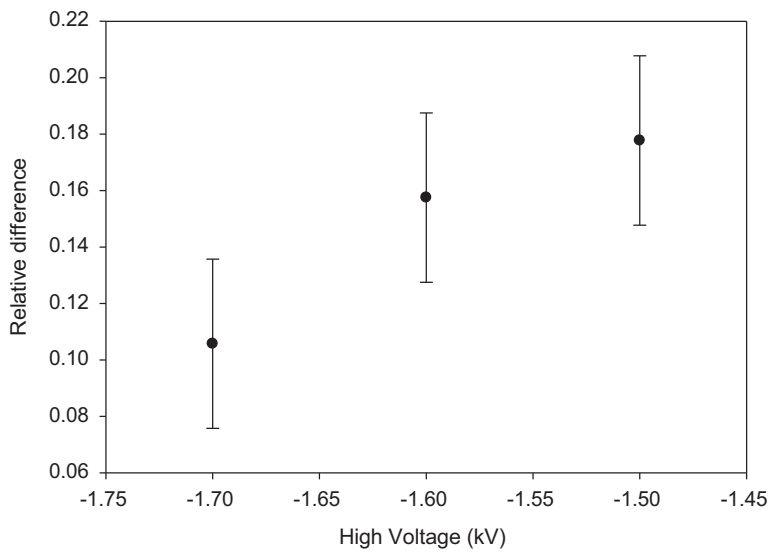

Fig. 8. The relative difference between the number of double and triple coincidences as a function of $\mathrm{HV}$ on alpha detector at threshold value on $\alpha \mathrm{CFD}=0$. Number of events was summed over the channels inside the time window " $I$ ". Random coincidences were subtracted.

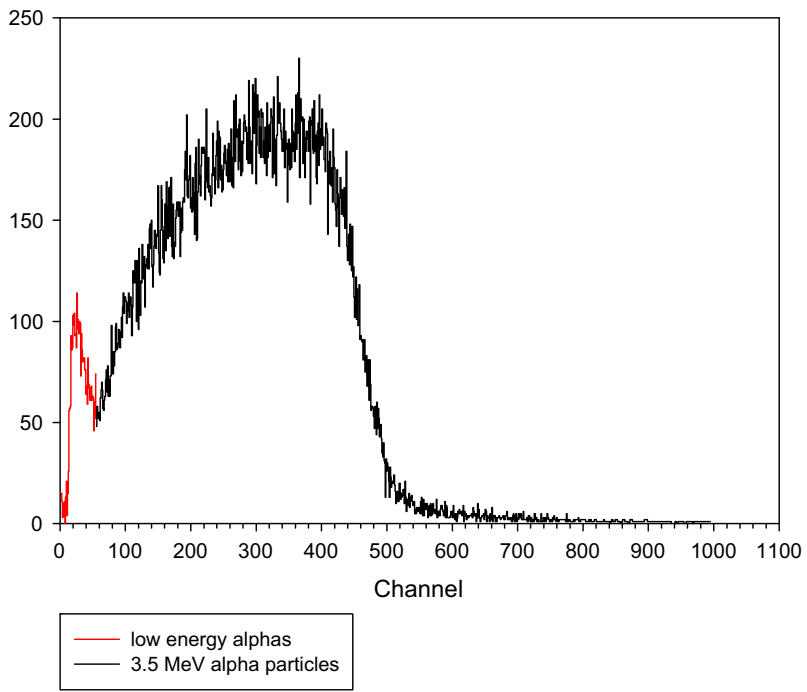

Fig. 9. Pulse height distributions for the neutron gated alpha detector. (For interpretation of the references to color in this figure, the reader is referred to the web version of this article.)

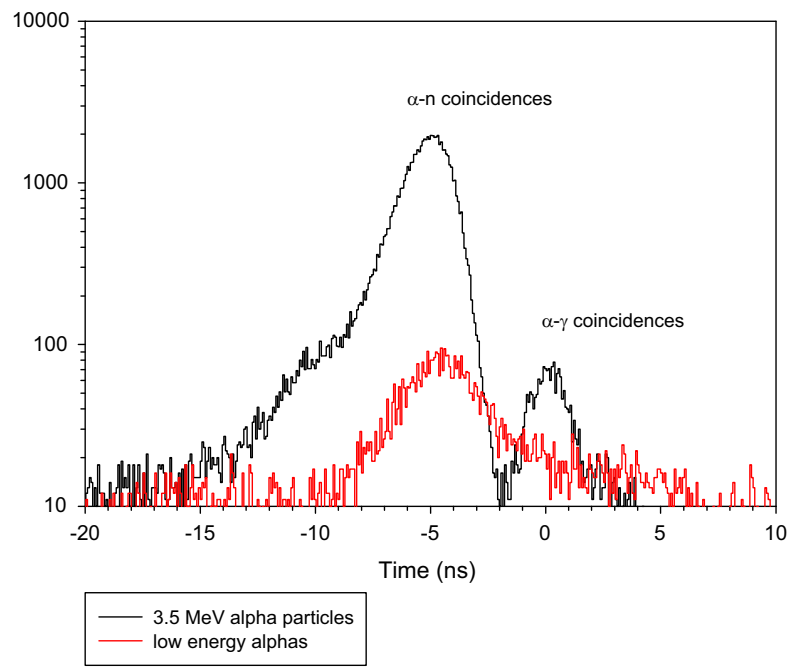

Fig. 10. Time spectrum between the alpha and neutron (NE213) detector. (For interpretation of the references to color in this figure, the reader is referred to the web version of this article.) 

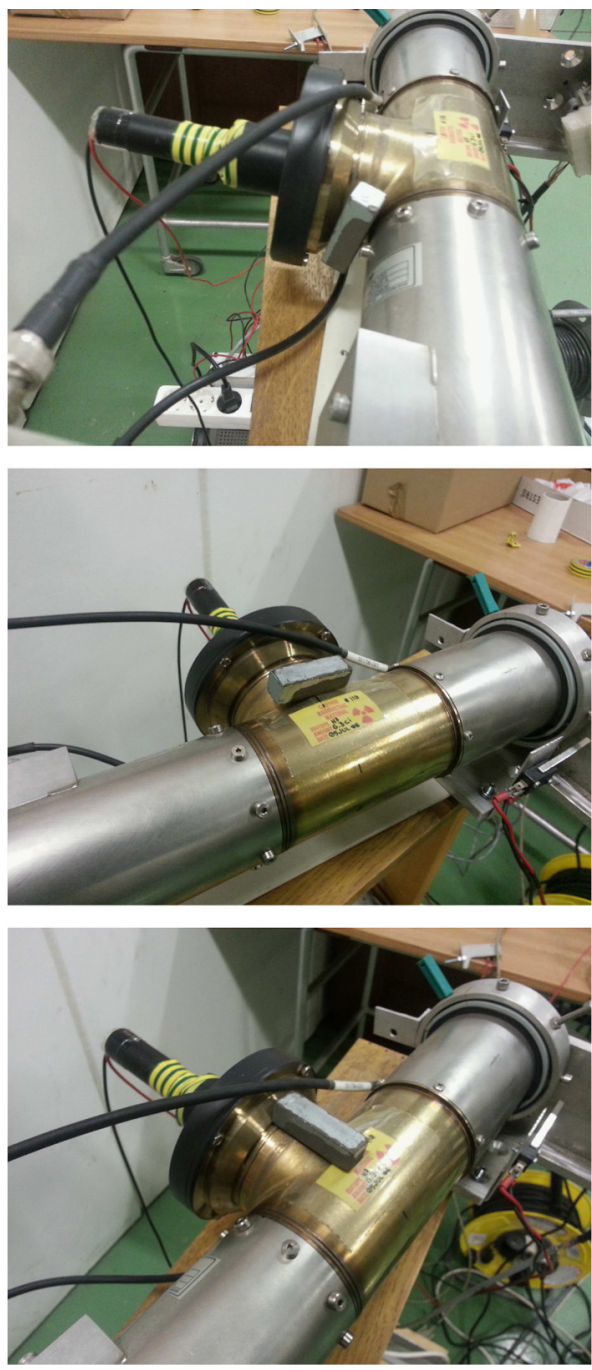

Fig. 11. Three different positions of permanent magnet close to the alpha detector. The positions are marked $\mathrm{a}, \mathrm{b}$ and $\mathrm{c}$ from up to down.

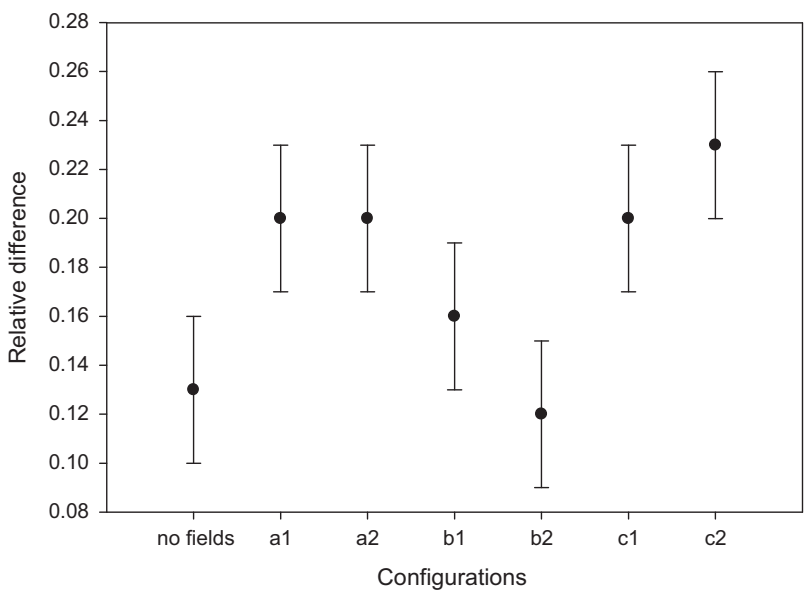

Fig. 12. The relative difference between double and triple coincidences in function of permanent magnet position. Number of events was summed over the channels inside the time window " $\Gamma$ ". Random coincidences were subtracted.

positions near the $\alpha$-particle detector (Fig. 11). At each position two measurements were performed, one for each magnet orientation ( $\mathrm{S}$ and $\mathrm{N}$ pole). It was assumed that under the influence of magnetic field $\alpha$-particles loose energy because of the longer spiral path to the detector and increased collision probability. The relative double/triple

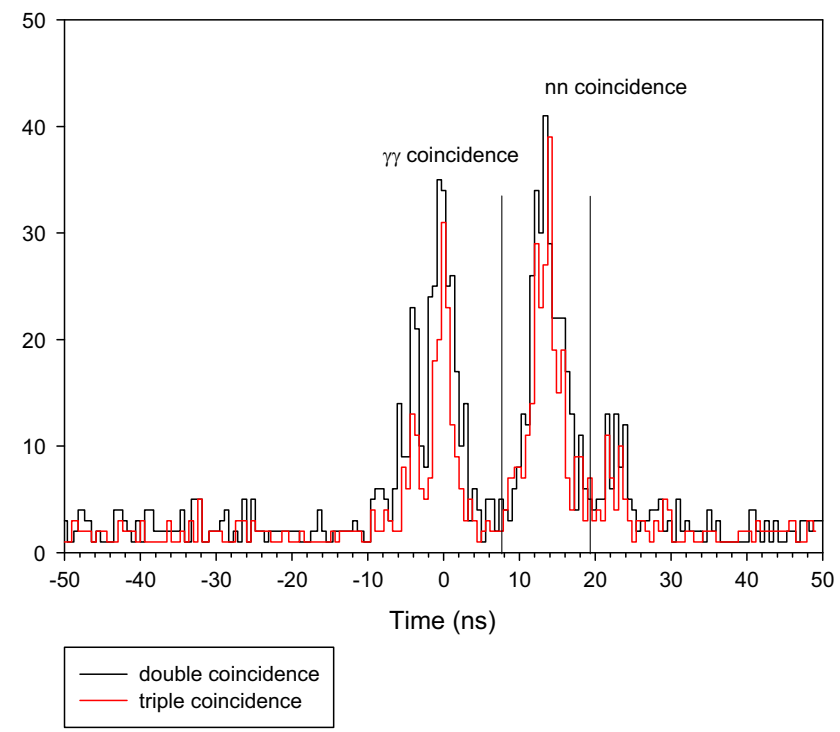

Fig. 13. Time spectra for events detected in the two neutron detectors. Double coincidences, $n-n$ (black) and triple coincidences, $n-n-\alpha$ (red) for target $\# 1$, marked with solid lines. (For interpretation of the references to color in this figure legend, the reader is referred to the web version of this article.)

coincidences difference as a function of permanent magnet position is shown in Fig. 12.

\subsection{Measurements with Cockcroft-Walton accelerator}

In order to obtain additional information on the associated $\alpha$-particle deficit, measurements have been performed with three different tritium targets. The active part of the targets was $6 \mathrm{~mm}$ in diameter and consisted of titanium $\left(0.95 \mathrm{mg} / \mathrm{cm}^{2}\right)$ deposited on copper backing ( $7 \mathrm{~mm}$ in diameter and $0.3 \mathrm{~mm}$ thickness). The activity at the beginning of the targets lifetime was $\sim 30 \mathrm{GBq} / \mathrm{cm}^{2}$. The titanium-tritiated target thickness was around $2 \mu \mathrm{m}$. Tritium targets have had different hours of previous usage and therefore different amounts of available tritium and implanted deuterium. Namely, there is a possibility that as a consequence of ${ }^{2} \mathrm{H}(\mathrm{d}, \mathrm{n})^{3} \mathrm{He}$ nuclear reaction $\left(E_{\mathrm{n}}=2.5 \mathrm{MeV}\right.$ and $\left.{ }^{3} \mathrm{He} \approx 0.8 \mathrm{MeV}\right)$ and insufficient time resolution of the neutron detectors, the excess of double $n-n$ coincidences is produced by $2.5 \mathrm{MeV}$ neutrons being counted in addition to $14 \mathrm{MeV}$ neutrons. ${ }^{3} \mathrm{He}$ of $0.8 \mathrm{MeV}$ energy could eventually pass through the YAP detector protection layer to be detected with its minimal energy. This effect could explain the observed decrease of $\alpha$ particle deficit by lowering the discriminator threshold, in addition to the effect of the background increase due to electronic noise which worsens as the signal approaches the low energy detector limit.

The measurements performed with three tritium targets under the same conditions in terms of alpha detector voltage $(V=-1.7 \mathrm{kV})$ and $\alpha$ CFD threshold $(0)$ resulted in very variable results not related to the target usage time. No peaks due to $2.5 \mathrm{MeV}$ neutrons were observed in the time spectra. The following results for double-triple/ double coincidences were obtained when putting the window for counting $n-n$ coincidences on $14 \mathrm{MeV}$ neutrons: target $\# 1=20 \pm 2 \%$, target $\# 2=27 \pm 4 \%$ and target $\# 3=12 \pm 3 \%$ (Fig. 13). It was noticed that this number can be altered by varying the position of deuteron beam on the tritium target.

\section{Discussion}

The first neutron detector, NE213, was located exactly in the middle of the tagged neutron cone. Since its diameter of $7.62 \mathrm{~cm}$ is smaller than the HWFM of the neutron cone $50 \mathrm{~cm}$ distant from 
the neutron source $(\mathrm{HWFM}=15 \mathrm{~cm})$, it is expected that every neutron hitting the NE213 has its associated alpha particle detected by the $\alpha$-particle detector. Neutrons which are not tagged can be scattered from the heavy elements in the surroundings and make some contributions to the double coincidence counts, which can explain part of the difference between the double and triple coincidence rate. However, contribution from the scattering should not be correlated with the threshold/HV voltage set on the $\alpha$-particle detector as experimentally observed. The later can be explained by the $\alpha$-particles energy loss.

Since we have tested only the one type of the $\alpha$-particle detector, the YAP scintillator with the rather bad energy resolution, it would be of interest to repeat these measurements with some other kind of $\alpha$-particle detector which has better energy resolution.

It should be mentioned that, contrary to our conclusions, Angelone et al. [7] reported that the absolute neutron yield of the $14 \mathrm{MeV}$ generators was routinely measured by means of the associated alpha-particle method with a silicon barrier detector, SSD. A complementary calibration procedure for validating the SSD results, based on the use of fission chambers and the activation technique, was also reported. An accurate analysis of the system has been performed via the Monte Carlo neutron and photon MCNP transport code. A detailed model of the neutron source that included ion slowing down has been inserted into the MCNP code to permit a numerical calibration of the neutron source for comparison with the experimental results. The resulting agreement among the various methods was very good considering the uncertainties, and an accuracy of $\pm 2 \%$ was claimed to be achieved for the measurement of the $14 \mathrm{MeV}$ neutron yield. The tritium target used in [7] is $4.4 \mu \mathrm{m}$ thick whereas our target used on Cockcroft-Walton accelerator were $\sim 2 \mu \mathrm{m}$ thick.

Hausladen et al. [8] used the experimental set-up similar to one described here. They noticed $10 \%$ loss of alpha particles, which they attributed to the alpha detector efficiency which was assumed to be $90 \%$ instead of expected $100 \%$, because of its special design resulting in the pulse height spectrum for mono-energetic $\alpha$-particles stretching down to zero height. In the case presented here (as shown in Fig. 9) the neutron-gated pulse height distributions for the $\alpha$-particle detector mounted on API-120 for $-1.6 \mathrm{kV}$ HV and zero thresholds is represented by a well-defined peak. Although broad, peak is shifted from the origin and only a small part of it (if any) is truncated by discriminator.

Alpha spectrum, shown on Fig. 9, has a peculiar shape, although it is in agreement with Fig. 7. It is shown (Figs. 9 and 10) that the lower (red) part of spectrum is shifted in time compared with the black part by $0.6 \mathrm{~ns}$, which correspond to the $\leq 2.9 \mathrm{MeV}$ alphas in red region and $3.5 \mathrm{MeV}$ in the black one. It is not clear why there is a hole between these two distributions of $\alpha$-particles. Someone would expect a broad $\alpha$-particle spectrum if there are energy losses. One possible explanation is a loss of energy inside the tritium target. Magnetic field can produce a vertical component of alpha particle speed, with respect to the main speed component, forcing the alpha particle to follow spiral curve in his way to the detector. It means that some alphas may go deeper to the tritium target instead to go directly out from the target. In addition, the $\alpha$-particle spectrum was acquired with zero threshold where lot of noise can produce a peculiar electronic artefact. In other words, the red part of spectrum may not be physical, but artificial electronic noise effect. Nevertheless, we think that the low energy component of the alpha spectrum indeed contains alpha particle that lost some energy in the tritium target, since we see that this component is at the good alpha-neutron position in the time spectrum of Fig. 10.

\section{Conclusions}

For correct cross section measurements of $14 \mathrm{MeV}$ neutron induced nuclear reactions, it is necessarily to know the neutron flux incident on the target. Since the neutron flux can be routinely measured by using the associated alpha particle technique (AAPT), it is necessarily to investigate the possible AAPT drawback.

In all measurements performed, a deficit of triple with respect to double coincidences has been observed. This deficit was smallest for $\alpha$ CFD threshold being 0 and for maximal allowed voltage on alpha detector $-1.7 \mathrm{kV}$ equaling $13 \pm 1 \%$. The number of non-detected $\alpha$-particles raised by increasing the threshold on $\alpha C F D$ and by decreasing the voltage on alpha detector. It was assumed that the alpha detector efficiency was approximately $100 \%$ and that missing $\alpha$-particles are due to the loss of energy during travel to the detector and consequently falling under the threshold of the $\alpha$ CFD discriminator. The $\alpha$-particles energy loss may be result of the collisions with other particles present in the neutron tube and/or in the tritium target. Magnetic fields present in the system worsen the situation since they are forcing $\alpha-$ particles to travel larger distances and undergo additional collisions on their toroidal path to the detector.

\section{References}

[1] V. Valkovic, D. Miljanic, P. Tomas, B. Antolkovic, M. Furic, Nuclear Instruments and Methods 76 (1969) 29.

[2] Le Tourneur, P., Dumont, J.L., Groiselle, C., Lacroix, J.S., Lefesvre, I., Lopez Jimenez, M.J., Paul, P., Poirrier, E., Mangeard, M., Vernet, B., Dardennes, C., Moutrousteguy, F. Thebault, F., Soudani, K., 2009: ULIS: A portable device for chemical and explosive detection, in: Proceedings of the International Topical Meeting on Nuclear Research Applications and Utilization of Accelerators, paper SM/EN-17 IAEA, Vienna, Austria.

[3] Sowerby, B.D., Cutmore, N.G., Liu, Y., Peng, H, Tickner, J.R., Xie, Y. and Zong C., Recent developments in fast neutron radiography for the interrogation of air cargo containers, in: Proceedings of the IAEA Conference, Paper: SM/EN-01, Vienna, 4-8 May 2009.

[4] V. Valković, D. Sudac, S. Blagus, K. Nađ, J. Obhođaš, B. Vekić, Nuclear Instruments and Methods in Physics Research B 263 (1) (2007) 119.

[5] Perot, B. et al. 2006: The EURITRACK project: development of a tagged neutron inspection system for cargo containers, in: Proceedings of the SPIE 6213, Orlando, Florida, 2006, pp. 621302-1,6.

[6] Mihalczo, J.T., Bingham, P.R., Blackston, M.A., Crye, J.M., Grogan, B.R., Hausladen, P.A., McConchie, S.M., Mullens, J. A., 2013. Fast-neutron imaging with API d-t neutron generators, in: (Ed.-in-chief: Barmakov, Yu. N.), Proceedings of the 2012 International Scientific and Technical Conference, All-Russia Research Institute of Automatics-VNIIA, Moscow, Russia, pp. 198-212.

[7] M. Angelone, M. Pillon, P. Batistoni, M. Martini, M. Martone, V. Rado, Review of Scientific Instruments 67 (1996) 2189-2196.

[8] P.A. Hausladen, J.S. Neal, J.T. Mihalczo, Nuclear Instruments and Methods in Physics Research B 241 (2005) (2005) 835. 